\title{
Deregulated miRNAs Contribute to Silencing of B-Cell Specific Transcription Factors and Activation of NF- $\kappa B$ in Classical Hodgkin Lymphoma
}

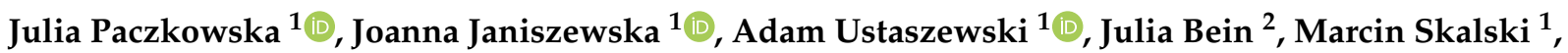 \\ Agnieszka Dzikiewicz-Krawczyk ${ }^{1}{ }^{(D}$, Natalia Rozwadowska $^{1}{ }^{(D)}$, Martin-Leo Hansmann ${ }^{2}$, Sylvia Hartmann $^{2}{ }^{\mathbb{D}}$ \\ and Maciej Giefing ${ }^{1, * \mathbb{D}}$
}

1 Institute of Human Genetics, Polish Academy of Sciences, 60-479 Poznan, Poland; julia.paczkowska@igcz.poznan.pl (J.P.); joanna.janiszewska@igcz.poznan.pl (J.J.); adam.ustaszewski@igcz.poznan.pl (A.U.); marcinskalski1993@gmail.com (M.S.); agnieszka.dzikiewicz-krawczyk@igcz.poznan.pl (A.D.-K.); natalia.rozwadowska@igcz.poznan.pl (N.R.)

2 Dr. Senckenberg Institute of Pathology, Goethe University Frankfurt, D-60590 Frankfurt, Germany; julia.bein@kgu.de (J.B.); martin-leo.hansmann@kgu.de (M.-L.H.); s.hartmann@em.uni-frankfurt.de (S.H.)

* Correspondence: maciej.giefing@igcz.poznan.pl; Tel.: +48-61-6579-138

Citation: Paczkowska, J.;

Janiszewska, J.; Ustaszewski, A.; Bein, J.; Skalski, M.; DzikiewiczKrawczyk, A.; Rozwadowska, N.; Hansmann, M.-L.; Hartmann, S.; Giefing, M. Deregulated miRNAs Contribute to Silencing of B-Cell Specific Transcription Factors and Activation of NF- $\kappa$ B in Classical Hodgkin Lymphoma. Cancers 2021, 13, 3131. https://doi.org/10.3390/ cancers13133131

Academic Editor:

Elisabetta Abruzzese

Received: 14 May 2021

Accepted: 14 June 2021

Published: 23 June 2021

Publisher's Note: MDPI stays neutral with regard to jurisdictional claims in published maps and institutional affiliations.

Copyright: (c) 2021 by the authors. Licensee MDPI, Basel, Switzerland. This article is an open access article distributed under the terms and conditions of the Creative Commons Attribution (CC BY) license (https:/ / creativecommons.org/licenses/by/ $4.0 /)$.
Simple Summary: The role of transcriptionally deregulated miRNAs (microRNAs) in classical Hodgkin lymphoma (cHL) is still not fully understood. To address this issue, we have performed global miRNA expression profiling of commonly used cHL cell lines and we present a complete cHL miRNome (microRNome). Within this group, we identify miRNAs recurrently deregulated in cHL cell lines, and compare them to non-Hodgkin lymphoma cell lines and sorted normal CD77 ${ }^{+}$ germinal centre B-cells. Moreover, we show that several of the recurrently overexpressed miRNAs in cHL cell lines, and also primary microdissected HRS (Hodgkin and Reed-Sternberg) cells, target known B-cell-related transcription factors and NF- $\mathrm{kB}$ inhibitors. These findings provide evidence that deregulated miRNAs contribute to the loss of B-cell phenotype and NF- $\mathrm{KB}$ activation observed in this lymphoma.

Abstract: A hallmark of classical Hodgkin lymphoma (cHL) is the attenuation of B-cell transcription factors leading to global transcriptional reprogramming. The role of miRNAs (microRNAs) involved in this process is poorly studied. Therefore, we performed global miRNA expression profiling using RNA-seq on commonly used cHL cell lines, non-Hodgkin lymphoma cell lines and sorted normal $\mathrm{CD}^{+} 7^{+}$germinal centre B-cells as controls and characterized the cHL miRNome (microRNome). Among the 298 miRNAs expressed in cHL, 56 were significantly overexpressed and 23 downregulated $(p<0.05)$ compared to the controls. Moreover, we identified five miRNAs (hsa-miR-9-5p, hsa-miR-243p, hsa-miR-196a-5p, hsa-miR-21-5p, hsa-miR-155-5p) as especially important in the pathogenesis of this lymphoma. Target genes of the overexpressed miRNAs in cHL were significantly enriched $(p<0.05)$ in gene ontologies related to transcription factor activity. Therefore, we further focused on selected interactions with the SPI1 and ELF1 transcription factors attenuated in CHL and the NF-kB inhibitor TNFAIP3. We confirmed the interactions between hsa-miR-27a-5p:SPI1, hsa-miR-330-3p:ELF1, hsa-miR-450b-5p:ELF-1 and hsa-miR-23a-3p:TNFAIP3, which suggest that overexpression of these miRNAs contributes to silencing of the respective genes. Moreover, by analyzing microdissected HRS cells, we demonstrated that these miRNAs are also overexpressed in primary tumor cells. Therefore, these miRNAs play a role in silencing the B-cell phenotype in cHL.

Keywords: classical Hodgkin lymphoma; microdissection; miRNA; loss of B-cell phenotype; NF-кB; B-cell transcription factors 


\section{Introduction}

Global epigenetic reprograming, mediated by alterations in DNA methylation, distinguishes classical Hodgkin lymphoma (cHL) from not only normal germinal centre B (GCB) cells, but also from other germinal centre derived B-cell lymphomas [1]. Aberrant DNA methylation is crucial for the survival of the neoplastic cells of cHL-the Hodgkin and Reed-Sternberg (HRS) cells-as shown by the regression of a relapsed metastatic cHL in a patient treated with the demethylating agent 5-azacytidine for myelodysplastic syndrome, or by the enhanced efficacy of immune checkpoint inhibitors after therapy using demethylating agents [2,3]. The canonical mechanism through which epigenetic alterations contribute to neoplastic disease is the hypermethylation of the promoter regions of tumor suppressor genes (TSGs), leading to their transcriptional silencing or the activation of oncogenes by loss of DNA methylation. However, in CHL, in addition to the usual hypermethylation of TSG promoters, DNA hypermethylation was reported to contribute to the loss-of-B-cell phenotype of HRS cells by silencing the B-cell specific transcription factors [4,5]. In this context, most studies focus exclusively on DNA methylation; in contrast, the role of the other epigenetic mechanism, namely miRNA in the regulation of B-cell specific transcription factors in the pathogenesis of cHL has remained largely understudied. However, the importance of miRNAs in normal B-cell development shown in many studies allows us to speculate on the putative role of miRNA deregulation in silencing B-cell specific transcription factors and the consequent loss-of-B-cell identity of HRS cells [6-9].

Therefore, in this study, we performed miRNA expression profiling using small RNA sequencing (RNA-seq) experiments in cHL cell lines, sorted GCB cells and non-Hodgkin lymphoma (NHL) cell lines. These data were used to (i) establish a complete cHL-specific miRNA expression profile and (ii) identify the group of miRNAs deregulated in cHL. Moreover, using in silico enrichment analysis and experimental validation, we identified several deregulated miRNAs involved in the attenuation of B-cell specific transcription factors and the constitutive activity of NF-kB in this lymphoma. Our study suggests that, in addition to DNA methylation, deregulation of miRNAs is another epigenetic process leading to the loss B-cell phenotype.

\section{Materials and Methods}

\subsection{Lymphoma Cell Lines, GCB Cells and Primary cHL Biopsies}

Three cHL cell lines (U-HO1, SUP-HD1, HDLM-2), 10 NHL cell lines (Burkitt lymphoma: Raji, Ca46, Daudi, Namalwa, Ramos; Diffuse Large Cell Lymphoma: OCI-LY1, OCI-LY3, OCI-LY7, SU-DHL-6; B Cell Lymphoma: Val) were obtained from the DSMZ resource center (Braunschweig, Germany). Moreover, four cHL cell lines (L-428, L-540, L-1236, KM-H2) obtained from collaborating partners were STR-typed to confirm their identity. The GCB6-16 cell line derived from immortalized GCB cells was a kind gift from Prof. Ralf Küppers (University of Duisburg-Essen, Essen, Germany) and Prof. Martin-Leo Hansmann (University of Frankfurt/Main, Frankfurt/Main, Germany). Cell lines were grown in RPMI medium supplemented with 10\% FBS (U-HO1, SUP-HD1, L-428, L-1236, KM-H2, RAJI, CA-46, DAUDI, NAMALWA, VAL) or 20\% FBS (L-540, HDLM-2, RAMOS, SU-DHL-6, OCI-LY3, GCB6-16). In contrast, the OCI-LY1 and OCI-LY7 cell lines were grown in IMDM medium (Thermo Fisher Scientific, Waltham, MA, USA) supplemented with $20 \%$ FBS.

Normal CD77 ${ }^{+} \mathrm{GCB}$ cells were purified from fresh tonsils obtained from tonsillectomies of chronic hyperplastic tonsillitis using magnetic activated cell sorting (MACS; Miltenyi Biotech, Bergisch Gladbach, Germany), as reported previously [10]. Primary HRS cells and adjacent bystander cells used as controls were microdissected from frozen lymph node sections HE (hematoxylin and eosin) stained of cHL patients using the PALM Robot MicroBeam laser microdissection system (PALM, Bernried, Germany) and pooled into groups of 1000 cells. The study was approved by the local ethics committee of the Goethe University Hospital (157/17 from 6 April 2017). 


\subsection{NGS Based miRNA Profiling}

Total RNAs from cell lines were isolated using the Trizol reagent (Thermo Fisher Scientific, Waltham, MA, USA) based on the Chomczyński method [11]. RNAs from sorted GCB cells and microdissected HRS cells were isolated using miRNeasy Mini Kit (Qiagen, Hilden, Germany). RNA quality and quantity was measured using the NanoDrop ND100 (Thermo Fisher Scientific, Waltham, MA, USA) and analyzed using the Agilent Bioanalyzer (Agilent, Santa Clara, CA, USA).

Total RNAs were shipped to the BGI company (Shenzhen, China) for small RNA sequencing. The NEBNext ${ }^{\circledR}$ Small RNA Library Prep Set for Illumina ${ }^{\circledR}$ (New England Biolabs Ipswich, MA, USA) was used for library preparation and small RNA sequencing experiments were performed using the HiSeq 4000 platform (Illumina, San Diego, CA, USA) and the SE50 (single-end $50 \mathrm{bp}$ ) sequencing protocol. Quality control was performed using FastQC software (Andrews S. (2010). FastQC: a quality control tool for high throughput sequence data. Available online at: http:/ / www.bioinformatics.babraham.ac.uk/projects / fastqc, accessed on 14 May 2021) showing very high quality of the reads (Phred quality score above Q30). The obtained reads have been mapped to reference genome (GRCh37) using Bowtie (http:/ / bowtie-bio.sourceforge.net/index.shtml, accessed on 31 March 2016) software, and the miRNA quality has been estimated with the CAP-miRSeq (http:// bioinformatics.mayo.edu/research/cap-mirseq/, accessed on 31 March 2016) package. Further processing of the data including filtration, normalization and statistical calculations was performed using the edgeR package (Bioconductor) (PMID: 19910308).

Two NGS experiments were performed. The first included cHL $(n=7)$ and NHL cell lines $(n=10)$ and the second included cHL $(n=3)$ and GCB samples $(n=10)$. We used counts per million (CPM) as a normalized determinant of miRNA expression. The CPM values of $\geq 10$ in at least $3 / 7 \mathrm{cHL}$ cell lines were regarded as indicative for the expression of a particular miRNA. Therefore, the miRNAome of $\mathrm{cHL}$ includes all detected miRNAs in the seven cHL cell lines fulfilling this criterion.

To identify miRNAs upregulated in $\mathrm{cHL}$, we selected miRNAs $(\log \mathrm{FC}>1.5 ; p<0.05)$ separately between (i) cHL and NHL and between (ii) cHL and GCB (differential expression analysis was performed using edgeR (PMID: 19910308)). Only miRNA expressed at least in 3/7 cHL were included. Similarly, for the miRNAs downregulated in CHL, we selected miRNAs (log FC $<-1.5 ; p<0.05$ ) separately between (i) cHL and NHL and between (ii) cHL and GCB. Only miRNAs expressed at least in 5/10 NHL and 5/10 GCB were included. With this filtering method, we received two sets of differently expressed miRNAs (cHL vs. NHL) and (cHL vs. GCB). By merging these two sets of miRNAs deregulated in $\mathrm{cHL}$, we created a common set of deregulated miRNAs in $\mathrm{cHL}$.

\subsection{Real-Time qPCR Based miRNA Expression Analysis}

The cDNA templates for real-time qPCR analyzes were synthesized from $10 \mathrm{ng}$ of total RNA using the TaqMan ${ }^{\mathrm{TM}}$ Advanced miRNA cDNA Synthesis Kit (Thermo Fisher Scientific, Waltham, MA, USA) according to supplier's protocol. In detail, poly(A) tailing was added to miRNAs followed by adapter ligation and the universal reverse transcription. Lastly, cDNA was amplified with universal forward and reverse primers.

Real-time qPCR reactions were performed in triplicate using the Bio-Rad CFX96 RealTime PCR System (Bio-RAD, Hercules, CA, USA) with TaqMan ${ }^{\text {TM }}$ Fast Advanced Master Mix (Thermo Fisher Scientific, Waltham, MA, USA ) and the TaqMan ${ }^{\mathrm{TM}}$ Advanced miRNA Assays (Thermo Fisher Scientific, Waltham, MA, USA) according to the protocol provided by the manufacturer (Thermo Fisher Scientific, Waltham, MA, USA). Using the BioRad Genex software (Bio-RAD, Hercules, CA, USA), the expression of particular miRNAs was calculated in relation to the miR-191-5p and miR-361-5 reference miRNAs, or in relation to the miR-let-7g and miR-361-5p reference miRNAs in the case of the real-time qPCR performed in microdissected HRS cells (Table S1). The chosen reference miRNAs showed stable expression across analyzed cell lines based on the small RNA-seq data. 


\subsection{Identification of Putative Target Genes of the cHL Deregulated miRNAs}

We used the Targetscan (http:/ / www.targetscan.org, accessed on 31 July 2017) prediction tool to identify putative target genes to be regulated (miRNA-mRNA interaction) by the two groups of miRNAs, the overexpressed and the downregulated in cHL. Target mRNA genes harboring a respective 8-mer and/or 7mer-m8 miRNA binding site in their $3^{\prime}$ UTR regions with a weighted context score below -0.5 were selected in each group. The two groups of target genes were analyzed for enrichments in biological process (gene ontology (GO) analysis) using the PANTHER database (http:/ / pantherdb.org/, accessed on 31 July 2017), the STRING database (http:/ / string-db.org, accessed on 31 July 2017) and the DAVID database (https:/ / david.ncifcrf.gov, accessed on 14 May 2021).

\subsection{Validation of miRNA Target Genes}

\subsubsection{Vector Preparation}

Fragments of the $3^{\prime}$ UTR regions of selected genes (ELF1, SPI1, TNFAIP3), containing binding sites for the analyzed miRNAs were synthesized (Genomed, Warsaw, Poland) and cloned into the pmirGLO Dual-Luciferase miRNA Target Expression Vector (Promega, Madison, WI, USA) followed by JM109 competent cells transformation (Promega, Madison, WI, USA). Wild type (WT) or mutant (MUT) variants of miRNA binding sites located in the $3^{\prime}$ UTRs were designed, as proposed by Mets et al. [12]. WT constructs contained miRNA binding sites of the respective $3^{\prime}$ UTR flanked with $30+/-\mathrm{bp}$. For MUT oligonucleotides, point mutations in the respective miRNA binding sites were introduced to diminish the putative interaction between the miRNA and the $3^{\prime} \mathrm{UTR}$ region (Table S2). Vectors were purified using PhasePrep BAC DNA Kit (Sigma-Aldrich, St. Louis, MO, USA) and verified by Sanger sequencing.

\subsubsection{Luciferase Reporter Assay}

Validation of the interactions between selected genes and miRNAs was performed in the HEK 293T cells. At least two independent transfections, in three technical repetitions were performed with jetPRIME DNA/siRNA (Polyplus-transfection SA, Illkirch, France) reagents as follows:

- In total, $500 \mathrm{ng}$ of vector containing the $3^{\prime} \mathrm{UTR}$ WT sequence $+50 \mu \mathrm{M}$ of the analyzed miRNA mimic (mirVana ${ }^{\circledR}$ miRNA mimic, Invitrogen, Waltham, MA, USA);

- In total, $500 \mathrm{ng}$ of vector containing the $3^{\prime} \mathrm{UTR}$ WT sequence $+50 \mu \mathrm{M}$ of the mimic negative control (mirVana ${ }^{\mathrm{TM}}$ miRNA Mimic, Negative Control \#1, Invitrogen, Waltham, MA, USA);

- In total, $500 \mathrm{ng}$ of vector containing the $3^{\prime} \mathrm{UTR}$ MUT sequence $+50 \mu \mathrm{M}$ of the analyzed miRNA mimic (mirVana ${ }^{\circledR}$ miRNA mimic, Invitrogen, Waltham, MA, USA);

- In total, $500 \mathrm{ng}$ of vector containing the $3^{\prime} \mathrm{UTR}$ MUT sequence $+50 \mu \mathrm{M}$ of the mimic negative control (mirVana ${ }^{\mathrm{TM}}$ miRNA Mimic, Negative Control \#1, Invitrogen, Waltham, MA, USA).

Cell lysis was performed with Dual-Glo Luciferase Assay System, (Promega, Madison, WI, USA), $24 \mathrm{~h}$ after transfection. The bioluminescence signal of firefly luciferase was measured using the GloMax ${ }^{\circledR} 96$ Microplate Luminometer (Promega, Madison, WI, USA) in reference to Renilla luciferase.

\subsection{MiRNA Overexpression}

The insert containing the miR-23a + mir-27a cluster was prepared by PCR amplification. Mature sequences of these miRNAs are encoded only $69 \mathrm{bp}$ apart and were therefore amplified together. Primers specific for the genomic sequence harboring the pre-miRNA hairpins, including approximately 100-250 nt flanking sequence on each site, were designed as described previously by Kluiver et al. [13] (Table S1). The PCR products with cohesive ends were cut with restriction enzymes (EcoRI and BamHI), purified and directly cloned into the pCDH-CMV-MCS-EF1 $\alpha$-GreenPuro vector (SBI, Palo Alto, CA, 
USA). The control empty vector was a kind gift from Dr Monika Drobna-Sledzinska (Institute of Human Genetics, Polish Academy of Sciences, Poznan, Poland). The vectors were packed into lentiviral particles in HEK 293T cells using jetPRIME transfection reagent (Polyplus-transfection SA, Illkirch, France). The lentiviral particles were harvested $48 \mathrm{~h}$ after transfection. Three independent transductions were performed with the vector carrying the respective miRNA sequences as well as by the empty vector. The expression of the GFP-puromycin resistance fusion gene from the vector was used for GFP/drug selection of the transduced cells. Efficiency of transduction was analyzed by flow cytometry and the expression construct harboring the respective miRNAs was validated by real-time qPCR with TaqMan ${ }^{\circledR}$ Advanced miRNA Assay prior to Western blot $(\mathrm{Wb})$ analyses.

\subsection{Western Blots}

Total protein was extracted from the transduced cell lines using RIPA buffer (SigmaAldrich, St. Louis, MO, USA) with a protease inhibitor cocktail (BioShop, ON, Canada) and the protein concentration was measured using the Bradford assay (Bio-Rad, Hercules, CA, USA). Western blots were conducted according to the standard procedure using Mini Protean system (Bio-Rad, Hercules, CA, USA) as described previously [14]. Anti-TNFAIP3 (A20) (59A426, Thermo Fisher Scientific, Waltham, MA, USA, 1:1000) and anti-GAPDH (ab9485, Abcam, Cambridge, United Kingdom, 1:5000) antibodies were used. Proteins were visualized by the Clarity Western ECL Substrate (Bio-Rad, Hercules, CA, USA). The images were scanned and analyzed using the ChemiDoc XRS+ System (Bio-Rad, Hercules, CA, USA). Relative TNFAIP3 expression was analyzed in reference to GAPDH using the Image Lab software (Bio-Rad, Hercules, CA, USA). The full Western Blots can be found at Figure S2.

\section{Results}

3.1. The cHL miRNome and miRNAs Deregulated in $\mathrm{cHL}$

In order to characterize the miRNAome of cHL we performed small RNA sequencing using the HiSeq 4000 Illumina platform (Illumina, San Diego, CA, USA) on seven cHL cell lines. Together, we detected expression of 298 miRNAs in at least three out of the seven analyzed cHL cell lines (Table S3). These miRNAs present the complete cHL miRNAome established in this study, which is, to the best of our knowledge, the most detailed NGSbased miRNA profile of cHL cell lines published so far. The mean expression of the identified miRNAs in the seven cHL cell lines was 3335 CPM (range 8-295 760 CPM). The top five most abundantly expressed miRNA in cHL were hsa-miR-21-5p and hsa-miR$155-5 \mathrm{p}$, both with proven oncogenic roles in cHL, but also members of the let-7 family hsa-let-7f-5p and hsa-let-7a-5p and hsa-miR-92a-3p. The mean expression of the top five miRNA was $128645 \mathrm{CPM}$, which is $>30$ times higher than the average expression of the identified miRNAs.

As our main goal was to identify miRNAs deregulated exclusively in cHL we then compared the cHL profiles with profiles of controls that included 10 NHL samples and 10 GCB samples analyzed using the same platform and parameters. By this approach, we found 56 miRNAs to be overexpressed and 23 downregulated in $\mathrm{CHL}$, compared against both control groups (Figure 1A,B). This group of 79 miRNAs contained putative oncomiRs and tumor suppressor miRNAs that may be involved in the pathogenesis of $\mathrm{cHL}$ and may contribute to the unique phenotype of HRS cells. 


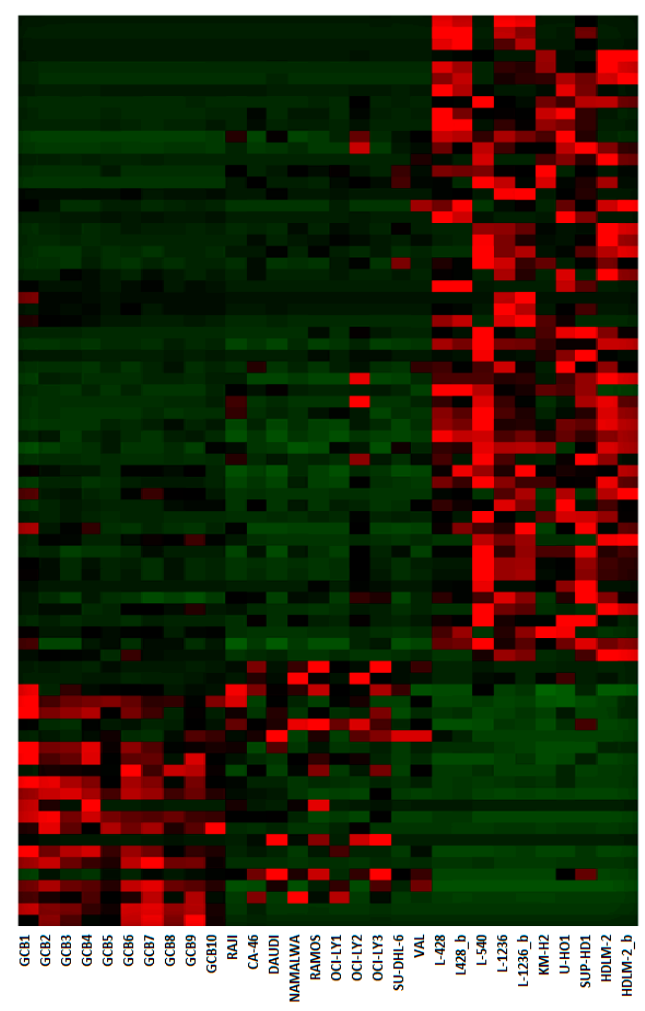

(A)
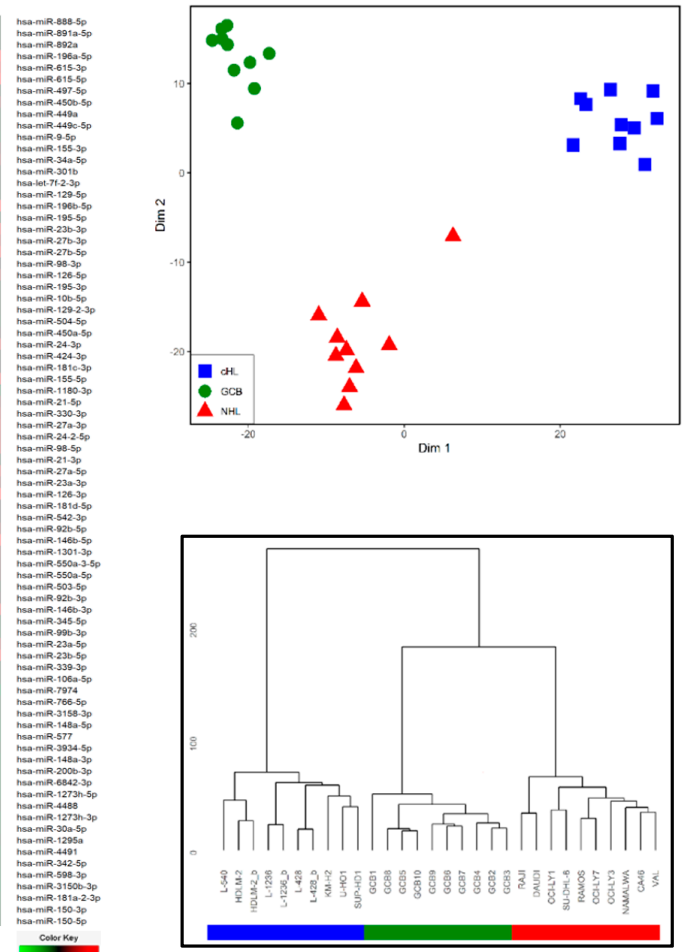

(B)
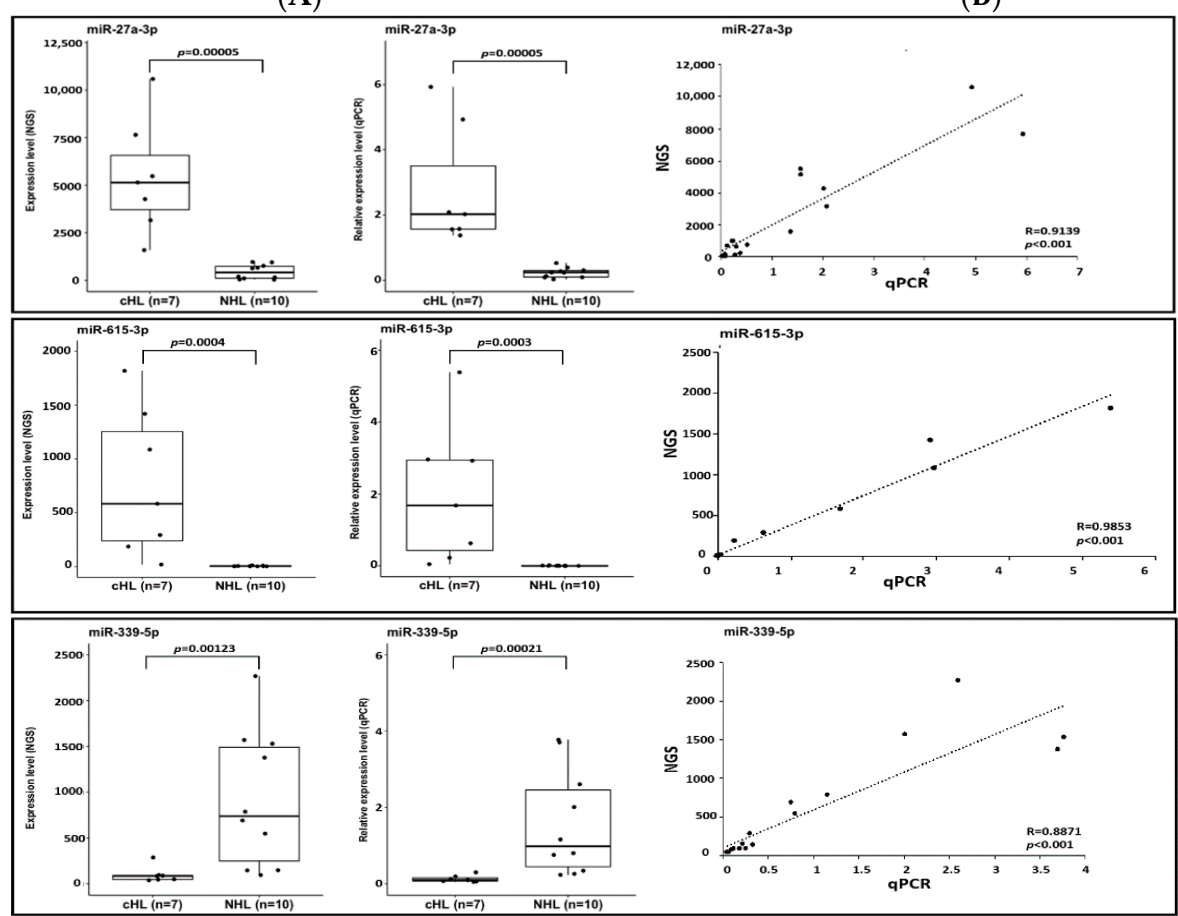

(C)

Figure 1. (A) Expression heat-map of the 79 miRNAs deregulated exclusively in cHL. GCB-germinal center B-cell pools $(n=10)$ are followed by non-Hodgkin lymphoma cell lines $(n=10)$ and cHL cell lines $(n=7)\left(\_b\right.$ after the cell line name indicates an experimental replication with modified library preparation for NGS. See the Methods section for details). (B) Multi-dimensional scaling (MDS) (input matrix was obtained using Canberra distance measure) of cHL, GCB and NHL samples based on expression of the 79 miRNAs deregulated exclusively in cHL (upper panel). Hierarchical clustering of the studied cohorts using Ward's method with the Canberra distance (lower panel). (C) Validation of NGS profiling. Expression of hsa-miR-615-3p, hsa-miR-27a-3p and hsa-miR-339-5p in cHL and NHL cell lines based on NGS and qPCR. 
To further validate the NGS-based profiles, we used real-time qPCR TaqMan ${ }^{\circledR}$ Advanced miRNA Assyas (Thermo Fisher Scientific, Waltham, MA, USA) for selected upregulated and downregulated miRNAs as a proof of principle analysis. Three deregulated miRNAs, namely miR-615-3p (upregulated in cHL), miR-27a-3p (upregulated in cHL) and miR-339-5p (downregulated w cHL) were analyzed (Figure 1C). Moreover, the expression of two miRNAs downregulated in cHL, namely miR-148a-3p and miR-148a-5p was validated in our previous manuscript [15]. The real-time qPCR-based expression results were highly convergent with those obtained by NGS, demonstrating the credibility of the small RNA sequencing data.

\subsection{Biological Processes Deregulated by miRNAs in $\mathrm{cHL}$}

We next focused our efforts on the identification of biological processes enriched in the group of genes targeted by the 79 miRNAs deregulated in cHL. In silico analysis using the TargetScan online tool (PMID: 26267216) resulted in the identification of 1298 genes as potential targets for the overexpressed miRNA in cHL, and 1649 genes as targets for the downregulated miRNAs.

These groups were then analyzed for functional enrichments independently using the PANTHER, STRING and DAVID databases. As GO analyses tend to generate large numbers of enriched biological terms, we compared only the top three biological processes identified for the groups of genes using each of the online tools. By this filtering, we identified the "DNA-binding transcription factor activity" (GO:0003700) $(p<0.03)$ biological process to be significantly enriched using all three databases for the upregulated miRNAs. Hence, this biological process is putatively attenuated in $\mathrm{CHL}$, as a consequence of the activity of the 56 miRNAs. This corresponds well with the known characteristic of HRS cells, which silence B-cell specific transcription factor activity. There was no enriched GO term found in all three databases for the group of downregulated miRNAs; however, one process, the "transcription regulatory region sequence-specific DNA binding" (GO:0000976), was returned by two out of three tools. Therefore, these putatively upregulated genes also seem to be involved to some extent in the regulation of transcription (Table 1).

Table 1. GO enrichment analysis for cHL deregulated miRNA target genes.

\begin{tabular}{|c|c|c|c|}
\hline \multirow[b]{2}{*}{ Top 3 GO Terms $(p<0.05)$} & \multicolumn{3}{|c|}{ GO Analysis of Target Genes of miRNAs Upregulated in cHL } \\
\hline & PANTHER Database & STRING Database & DAVID Database \\
\hline 1 & $\begin{array}{l}\text { DNA-binding transcription factor } \\
\text { activity, RNA polymerase } \\
\text { II-specific (GO:0000981) }\end{array}$ & $\begin{array}{l}\text { DNA-binding transcription } \\
\text { factor activity (GO:0003700) }\end{array}$ & $\begin{array}{l}\text { DNA-binding transcription } \\
\text { factor activity (GO:0003700) }\end{array}$ \\
\hline 2 & $\begin{array}{l}\text { DNA-binding transcription } \\
\text { factor activity (GO:0003700) }\end{array}$ & $\begin{array}{l}\text { molecular function regulator } \\
\text { (GO:0098772) }\end{array}$ & protein binding (GO:0005515) \\
\hline \multirow[t]{2}{*}{3} & $\begin{array}{l}\text { molecular function regulator } \\
\text { (GO:0098772) }\end{array}$ & $\begin{array}{l}\text { transcription regulator activity } \\
\text { (GO:0140110) }\end{array}$ & $\begin{array}{l}\text { regulation of transcription, } \\
\text { DNA-templated (GO:0006355) }\end{array}$ \\
\hline & \multicolumn{3}{|c|}{ GO analysis of target genes of miRNAs downregulated in $\mathrm{cHL}$} \\
\hline Top 3 GO terms $(p<0.05)$ & PANTHER database & STRING database & DAVID database \\
\hline 1 & $\begin{array}{l}\text { transcription regulatory region } \\
\text { sequence-specific DNA binding } \\
\text { (GO:0000976) }\end{array}$ & $\begin{array}{l}\text { DNA-binding transcription factor } \\
\text { activity, RNA polymerase } \\
\text { II-specific (GO:0000981) }\end{array}$ & $\begin{array}{l}\text { transcriptional activator activity, } \\
\text { RNA polymerase II core promoter } \\
\text { proximal region sequence-specific } \\
\text { binding (GO:0001077) }\end{array}$ \\
\hline 2 & $\begin{array}{l}\text { RNA polymerase II regulatory } \\
\text { region DNA binding } \\
\text { (GO:0001012) }\end{array}$ & $\begin{array}{l}\text { transcription regulatory region } \\
\text { sequence-specific DNA binding } \\
\text { (GO:0000976) }\end{array}$ & neuron migration (GO:0001764) \\
\hline 3 & $\begin{array}{l}\text { transcription regulatory region } \\
\text { DNA binding (GO:0044212) }\end{array}$ & $\begin{array}{l}\text { RNA polymerase II regulatory } \\
\text { region sequence-specific DNA } \\
\text { binding (GO:0000977) }\end{array}$ & $\begin{array}{l}\text { RNA polymerase II core promoter } \\
\text { proximal region sequence-specific } \\
\text { DNA binding (GO:0000978) }\end{array}$ \\
\hline
\end{tabular}


In light of these results, we further focused on deregulated miRNAs and their putative interactions with B-cell specific transcription factors SPI1 and ELF-1 attenuated in cHL $[5,16]$ and also the NF-kB inhibitor TNFAIP3 recurrently inactivated in this lymphoma [17]. We selected the putative hsa-miR-27a-5p:SPI1, hsa-miR-330-3p:ELF-1 (two miRNA binding sites were tested), hsa-miR-542-3p:ELF1, hsa-miR-450b-5p:ELF-1 and the hsa-miR-23a-3p:TNFAIP3 interactions for experimental testing (Table 2).

Table 2. miRNA:mRNA interactions tested experimentally.

\begin{tabular}{|c|c|c|c|c|c|c|c|}
\hline \multirow[b]{2}{*}{ MiRNA in cHL } & \multirow[b]{2}{*}{ MiRNA } & \multirow[b]{2}{*}{ Target Gene } & \multicolumn{3}{|c|}{$\begin{array}{c}\text { UTR miRNA Binding Site [Based on } \\
\text { TargetScan Database] }\end{array}$} & \multirow{2}{*}{$\begin{array}{c}\text { Interaction } \\
\text { Confirmed in } \\
\text { the Luciferase } \\
\text { Assay }\end{array}$} & \multirow{2}{*}{$\begin{array}{l}\text { Gene } \\
\text { Function }\end{array}$} \\
\hline & & & $\begin{array}{l}\text { Binding Sites } \\
\text { Conservation }\end{array}$ & $\begin{array}{l}\text { Number of } \\
\text { Binding Sites }\end{array}$ & $\begin{array}{c}\text { Characteristic } \\
\text { of Binding } \\
\text { Sites }\end{array}$ & & \\
\hline \multirow{5}{*}{ UPREGULATED } & $\begin{array}{l}\text { hsa-miR- } \\
\text { 27a-5p }\end{array}$ & SPI1 (PU.1) & $\begin{array}{l}\text { poorly } \\
\text { conserved }\end{array}$ & 1 & 7 mer-m8 & YES & $\begin{array}{c}\text { B-cell related } \\
\text { transcription } \\
\text { factor }\end{array}$ \\
\hline & $\begin{array}{l}\text { hsa-miR- } \\
\text { 330-3p }\end{array}$ & ELF-1 & $\begin{array}{l}\text { poorly } \\
\text { conserved }\end{array}$ & 2 & $\begin{array}{l}\text { 7mer-m8, } \\
7 \mathrm{mer}-\mathrm{m} 8\end{array}$ & YES & $\begin{array}{l}\text { B-cell related } \\
\text { transcription } \\
\text { factor }\end{array}$ \\
\hline & $\begin{array}{l}\text { hsa-miR- } \\
542-3 p\end{array}$ & ELF-1 & $\begin{array}{l}\text { poorly } \\
\text { conserved }\end{array}$ & 2 & $\begin{array}{l}\text { 7mer-A1, } \\
7 \text { mer-m8 }\end{array}$ & $\mathrm{NO}$ & $\begin{array}{l}\text { B-cell related } \\
\text { transcription } \\
\text { factor }\end{array}$ \\
\hline & $\begin{array}{l}\text { hsa-miR- } \\
450 \mathrm{~b}-5 \mathrm{p}\end{array}$ & ELF-1 & $\begin{array}{l}\text { poorly } \\
\text { conserved }\end{array}$ & 1 & 7mer-A1 & YES & $\begin{array}{l}\text { B-cell related } \\
\text { transcription } \\
\text { factor }\end{array}$ \\
\hline & $\begin{array}{l}\text { hsa-miR- } \\
\text { 23a-3p }\end{array}$ & TNFAIP3 & conserved & 1 & 8 mer & YES & $\begin{array}{c}\text { negative } \\
\text { regulator of } \\
\text { NF-kappaB }\end{array}$ \\
\hline
\end{tabular}

\subsection{Functional Validation of miRNA-mRNA Interactions for Selected Candidates}

We performed luciferase reporter assays to analyze whether the selected miRNAs indeed interact with the $3^{\prime}$ UTR regions of the genes attenuated in cHL. The strongest reduction $(34 \%, p<0.001)$ of the luciferase intensity was observed as a consequence of the interaction of the hsa-miR-23a-3p mimic with its respective binding site in the $3^{\prime}$ UTR region of the TNFAIP3 gene. However, a significant but less intensive signal reduction $(20 \%, p=0.0032)$ was observed also for the mutant $3^{\prime}$ UTR binding site, suggesting that the introduced mutations did not abolish the interaction entirely. Moreover, significant reduction of the luciferase intensity was observed for the hsa-miR-330-3p mimic and ELF1 (by $26 \%, p<0.001$ for the first and by $21 \%, p<0.01$ for the second binding site) and the hsamiR-450b-5p mimic and ELF1 (by 23\%, $p<0.001$ ). Interestingly, a co-transduction with the two ELF1 interacting mimics did not have a synergistic effect and resulted in the reduction of signal intensity by $25 \%(p<0.001)$. However, for hsa-miR-27a-5p:SPI1, we observed a less intensive reduction of the signal intensity (by $17 \%, p=0.05$ ) and no significant interaction for hsa-miR-542-3p and ELF1 (Figure 2). Hence, these results suggest that hsa-miR-330-3p, hsa-miR-450b-5p and hsa-miR-27a-5p, but not hsa-miR-542-3p, negatively regulate the respective transcription factors and that hsa-miR-23a-3p may contribute to constant activation of NF-kB in cHL.

These findings were further confirmed for the A20 (TNFAIP3) protein using Western blot. The abundance of the A20 protein was significantly reduced in the L428 $(p=0.0018)$ and the GCB6-16 ( $p=0.0083)$ cell lines after transduction with the mir-23a + mir-27a expression construct, as compared to the empty vector transduced cell lines (Figure 3). 

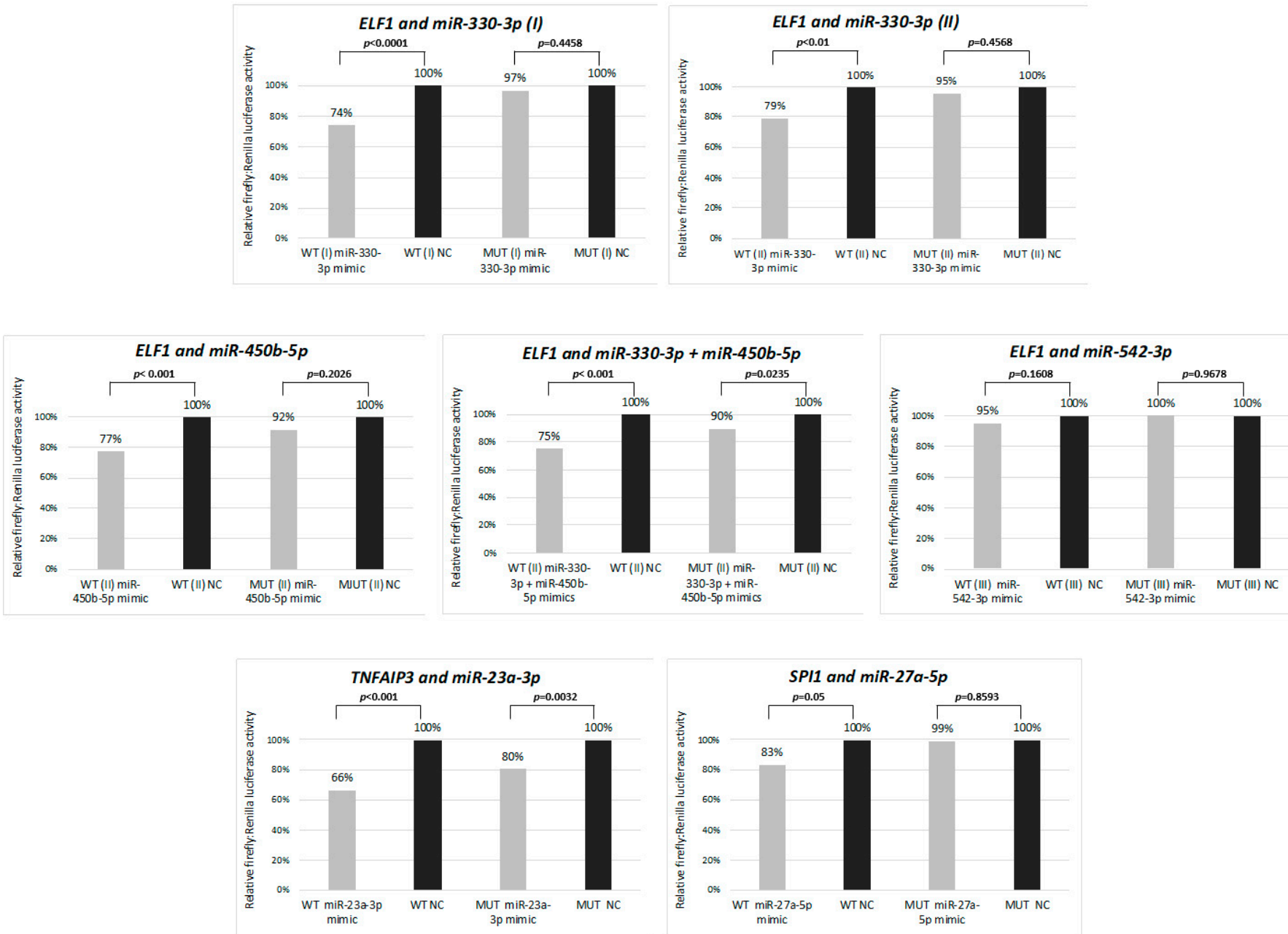

Figure 2. Interaction between selected miRNAs and their target genes (ELF1, TNFAIP3 and SPI1) validated by dual-luciferase reporter assay in HEK 293T cell line. WT: wild type 3'UTR, MUT: mutant $3^{\prime} U T R$, with point mutations in miRNA binding site. Mean from two independent experiments performed in triplicate is shown.

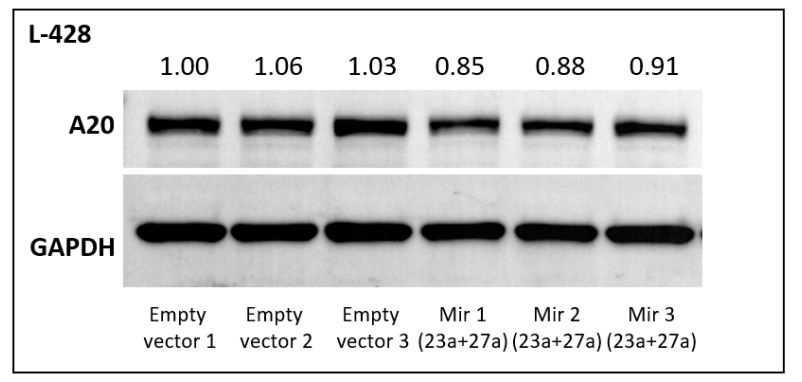

$p=0.0018$

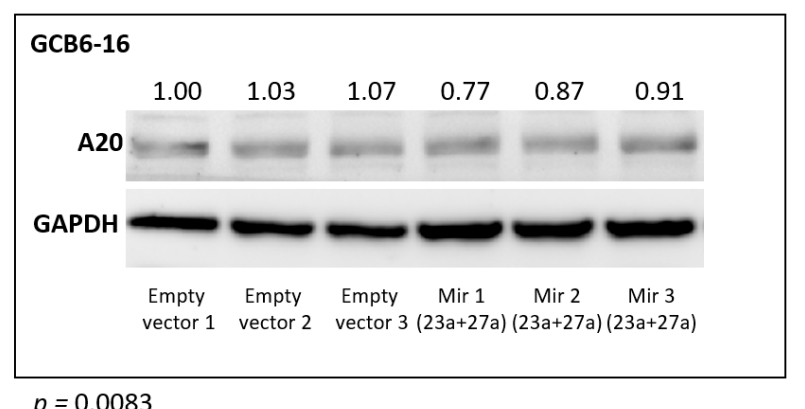

$p=0.0083$

Figure 3. Relative TNFAIP3 (A20) protein abundance after stable overexpression of hsa-mir-23a (23a) and hsa-mir-27a (27a) in L-428 and GCB6-16 cell lines, compared to cells transduced with the empty vector (three independent biological replications are shown).

\subsection{Validation of miRNA Expression in Microdissected Primary HRS Cells}

To confirm that the overexpression of hsa-miR-23a-3p, hsa-miR-330-3p, hsa-miR-450b$5 \mathrm{p}$ and hsa-miR-27a-5p observed in cHL cell lines is not a consequence of cell culturing, we used real-time qPCR molecular probes to analyze the expression of these miRNAs in microdissected HRS cell pools obtained from tumor samples $(n=10)$. The miRNA 
expression levels observed in HRS cells were compared with the expression in sorted GCB cell pools $(n=10)$ and NHL cell lines $(n=10)$. In line with the findings in cHL cell lines, in the microdissected HRS cells, hsa-miR-23a-3p and hsa-miR-27a-5p were significantly upregulated $(p<0.001$ and $p<0.05$ respectively), compared to both control groups. Similarly, hsa-miR-330-3p expression in the HRS cells was significantly upregulated $(p<0.03)$ versus GCB cells and elevated, albeit not significantly, versus the NHL cell lines $(p=0.126)$. Moreover, hsa-miR-450b-5p was significantly upregulated in the HRS cells $(p<0.001)$ compared to in the GCB pools (cHL and NHL cell lines were not included in miR-450b-5p expression analysis) (Figure 4 ).
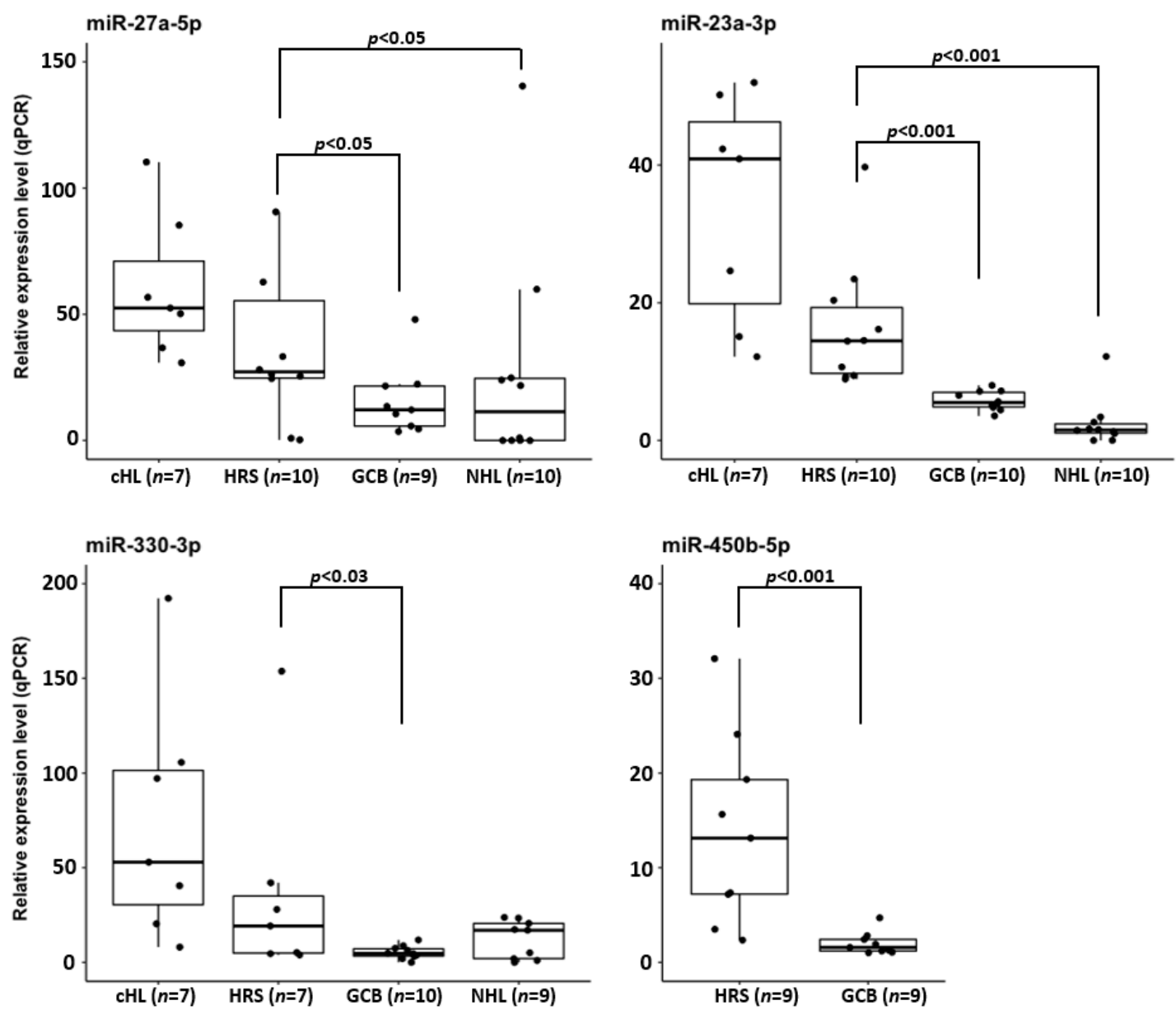

Figure 4. Overexpression of hsa-miR-27a-3p, hsa-miR-23a-3p and hsa-miR-330-3p in cHL cell lines and microdissected HRS cells from cHL cases in comparison to NHL cell lines, and CD77 ${ }^{+}$GCB cell pools and hsa-miR-450b-5p overexpression in microdissected HRS cells in comparison to $\mathrm{CD} 77^{+} \mathrm{GCB}$ cell pools.

Thus, primary HRS, similarly to cHL cell lines, overexpress these four miRNAs. Moreover, these data indicate that hsa-miR-27a-5p and hsa-miR-23a-3p overexpression might be a unique feature of cHL miRNome.

\section{Discussion}

In this study, we established a complete profile of 298 miRNAs expressed in seven commonly used cHL cell lines. Out of these, 126 miRNAs showed a mean expression level of at least $100 \mathrm{CPM}$, therefore these miRNAs probably exert biological effects in the cells. More specifically, we identified a group of 43 strongly expressed miRNAs (CPM $>1000$ and $<10,000)$ and a further 14 miRNAs with very high expression $(C P M>10,000)$ topped by hsalet-7f-5p, hsa-miR-155-5p (CPM > 100,000) and especially hsa-miR-21-5p (CPM 300,000) being the most abundantly expressed miRNA in the studied cHL cell lines. The two most 
abundantly expressed miRNAs in cHL, namely hsa-miR-21-5p and hsa-miR-155-5p, are particularly important in cHL pathogenesis, and were reported to be overexpressed in CHL in earlier studies [18-23]. Hsa-miR-21-5p was shown to act via targeting BTG2 and PELI1 mRNAs protecting the HRS cells from apoptosis [24] and hsa-miR-155-5p via targeting the tumor suppressor NIAM known to promote lymphomagenesis upon silencing $[18,25,26]$. Importantly, hsa-miR-155-5p contributes also to the constitutive activation of NFkB in HRS cells, as it was shown to interact with the inhibitor of NFKB kinase subunit epsilonIKBKE [21]. In addition, hsa-let-7f-5p was reported previously in cHL. Its exact function remains unknown; however, other studies suggested its role in silencing pro-apoptotic genes [27].

To systematically identify miRNAs deregulated exclusively in cHL cell lines, we compared their expression with NHL cell lines, as well as sorted GCB cells. This revealed 79 differentially expressed miRNAs in cHL compared to both control groups (56 upregulated and 23 downregulated). The expression profile of deregulated miRNAs in cHL established in our study shows only an average overlap with the profile reported by Yuan et al., who used a similar experimental approach based on small RNA-seq of cHL cell lines $(n=4)$ [28]. The authors reported overexpression of 55 miRNAs in cHL cell lines, out of which 25/55 (45\%) overlap with our study, and, similarly, the authors report 29 downregulated miRNAs out of which $9 / 29(31 \%)$ where found also in our study. However, it should be noted that we aimed to identify miRNAs deregulated exclusively in cHL therefore the expression of the candidate miRNAs was compared not only versus GCB cells but also other lymphomas which likely explains the relatively high discrepancy. Similarly, there is only minimal overlap with earlier reports on miRNA expression in primary microdissected HRS cells. For example, Van Vlierberghe reported the overexpression of 30 miRNAs in primary HRS cells using a miRNA RT kit, out of which five (17\%) were overexpressed, and none of the downregulated ones were identified in our analysis [20].

Despite the low overlap between the discussed studies, there emerges a common group of miRNAs found overexpressed in all three analyses in the cHL cell lines and primary HRS cells (for details see Figure S1). These include hsa-miR-9-5p, hsa-miR-24-3p, hsa-miR-196a-5p, hsa-miR-21-5p and hsa-miR-155-5p, although the latter two, with fold change $>3$ times in $\mathrm{cHL}$, were not classified by Yuan et al. as overexpressed, probably because of the strict selection criteria applied in the study. Four miRNAs from this list: hsamiR-9-5p [29,30], hsa-miR-21-5p [24], hsa-miR-24-3p [28] and hsa-miR-155-5p [18,25,26] have a proven oncogenic role in cHL. The role of hsa-miR-196a-5p in the pathogenesis of cHL requires further experimental validation, however it was recently reported that this miRNA promotes cell proliferation, migration and invasion of colorectal cancer. Moreover, what is especially interesting with regard to cHL pathology is that it acts via the inhibition of $\operatorname{I\kappa } B \alpha$, which could potentially contribute to the constant hyperactivity of NFkB [31].

As the putative target genes of the miRNAs found overexpressed in our study were significantly enriched in functions described as "DNA-binding transcription factor activity" (GO:0003700), we focused on the analysis of miRNAs that could potentially target transcription factors. We speculated that this mechanism likely contributes to the silencing of transcription factors attenuated in cHL that, as a consequence, leads to the loss of B-cell phenotype of HRS cells. In fact, several reports provide evidence that miRNAs are involved in regulation of all stages of mammalian B-cell development $[6,32,33]$. We confirmed experimentally that hsa-miR-330-3p, hsa-miR-450b-5p, hsa-miR-27a-5p and hsa-miR-23a-3p interact with $3^{\prime}$ UTR sites of ELF1 (miR-330-3p and miR-450b-5p), SPI1 (miR-27a-5p) and TNFAIP3 (miR-23a-3p). SPI1 (PU.1) and ELF1 encode transcription factors of known important B-cell development. More specifically, PU.1 has a proven role in the regulation of IgK transcription and rearrangement, as does ELF1 in the activation of several B-cell specific tyrosine kinases in mice that are important for proper BCR signaling [34,35]. Both factors are recurrently attenuated in cHL $[5,16]$. However, TNFAIP3 is a ubiquitin-modifying enzyme that targets the RIP1 protein for degradation. RIP1, in turn, is necessary to activate the IKK complex and subsequent NF- $\mathrm{kB}$ activation [36]. The importance of the miRNA:mRNA 
interactions described here is moreover stressed by the fact that these miRNAs were found to be overexpressed, not only in the cHL cell lines, but also in primary microdissected HRS cells.

These newly observed interactions were not previously linked to cHL pathogenesis. However, Guo et al. observed recently, using in vivo mouse and in vitro human cell models, that has-miR-23a-3p regulates inflammation and apoptosis by interacting with TNFAIP3 in atherosclerosis [37]. In line, this was the strongest interaction observed in our reporter assay experiments, suggesting that overexpression of this miRNA is an alternative and most probably a more subtle mechanism to the recurrent loss of function genomic alterations targeting TNFAIP3 in CHL [17]. We further validated this interaction on the protein level. The induced overexpression of hsa-miR-23a-3p in L428 (a cHL cell line showing genomic amplification of the TNFAIP3 gene locus and exceptionally strong expression of the protein) and GCB6-16 (immortalized GCB-cell derived cell line) resulted in the decrease of TNFAIP3 protein abundance.

Hsa-miR-23a-3p, together with hsa-miR-27a-5p, is expressed from the same lncRNA transcript (LOC284454) as the cHL oncogenic hsa-miR-24-3p, which is known to be upregulated in cHL cell lines [28]. However, in the GFP assay reported in the cited study, hsa-miR-23a-3p silencing in four tested cHL cell lines did not result in measurable growth retardation of the HRS cells. On one hand, this may suggest that hsa-miR-23a-3p is overexpressed by a passenger effect, together with the oncogenic miRNA encoded by the lncRNA. On the other hand, hsa-miR-23a-3p's inhibitory effect on TNFAIP3, as shown in our study and its manifestation on the cellular level in the tested cHL cell lines, is most probably hampered by various compound heterozygous loss-of-function mutations of TNFAIP3, such as W142STOP codon/del in L-1236, frameshift in amino acid 99/del in KM-H2, or frameshift in amino acid 174/del in HDLM-2 [17].

The involvement of hsa-miR-330-3p and hsa-miR-450b-5p in lymphomagenesis has not been reported so far. However, the oncogenic function of these miRNAs was demonstrated in several solid tumors including hsa-miR-330-3p in lung cancer [38] and breast cancer [39] and hsa-miR-450b-5p in colorectal cancer [40].

\section{Conclusions}

In conclusion, we present a detailed miRNome of the seven commonly used cHL cell lines and confirm the leading role of the hsa-miR-9-5p, hsa-miR-21-5p, hsa-miR-24-3p and hsa-miR-155-5p in cHL pathogenesis, as shown in previous studies. Moreover, we demonstrate four miRNAs that we found overexpressed in cHL cell lines, as well as microdissected HRS cells: hsa-miR-330-3p, hsa-miR-450b-5p, hsa-miR-27a-5p and hsa-miR-23a-3p to interact with respective mRNAs of TFs and TNFAIP3 attenuated in cHL pathogenesis. Together, these findings constitute a potential alternative silencing mechanism of B-cell specific TF and of NF-kB inhibitors in HRS cells.

Supplementary Materials: The following are available online at https: / www.mdpi.com/article / 10.3390/cancers13133131/s1, Figure S1: The overlap of deregulated miRNAs in cHL cell lines and primary HRS cells compared to NHL cell lines and GCB cells in previously published studies and this study. Columns represent miRNA expression profiles from the particular studies. Red and green colors indicate overexpressed and downregulated miRNAs, respectively, black color represents no significant change in miRNA expression in the respective study, Figure S2: Uncropped Figure 3, Table S1: Primer sequences used in the study; restriction sites are in lowercase, Table S2: Oligonucleotide sequences used in luciferase assays. MiRNA binding sites in 3'UTRs are underlined; introduced point mutations are in bold; restriction sites are marked in lowercase, Table S3: List of miRNAs expressed in cHL cell lines (miRNome). 
Author Contributions: Conceptualization, J.P. and M.G.; data curation, A.U.; formal analysis, J.P., A.U., A.D.-K., N.R., M.-L.H. and S.H.; funding acquisition, M.G.; investigation, J.P., J.J., J.B. and M.S.; methodology, J.P., J.J., J.B., A.D.-K. and N.R.; supervision, M.G.; visualization, J.P. and A.U.; writing-original draft, J.P., J.J., A.U. and M.G.; writing—review and editing, J.P., J.J., A.U. and M.G. All authors have read and agreed to the published version of the manuscript.

Funding: This research was funded by the Polish National Science Center through the 2014/14/M/ NZ2/00529 grant to MG and the 2019/32/T/NZ5/00441 Etiuda scholarship to JP. SH is supported by the German Research Foundation (DFG, HA6145/3-1). MG, ADz-K and NR received funding from the European Union's Horizon 2020 research and innovation programme under grant agreement No 952304.

Institutional Review Board Statement: The study was conducted according to the guidelines of the Declaration of Helsinki, and approved by the local ethics committee of the Goethe University Hospital (157/17 from 6 April 2017).

Informed Consent Statement: Informed consent was obtained from all subjects involved in the study.

Data Availability Statement: The data presented in this study are available on request from the corresponding author.

Acknowledgments: The authors would like to thank the Molecular Genetics Laboratory in Poznan (Laboratorium Genetyki Molekularnej) for performing cell line authentication and Natalia Soloch for excellent technical assistance.

Conflicts of Interest: The authors declare no conflict of interest.

\section{References}

1. Ammerpohl, O.; Haake, A.; Pellissery, S.; Giefing, M.; Richter, J.; Bálint, B.; Kulis, M.; Le, J.; Bibikova, M.; Drexler, H.G.; et al. Array-based DNA methylation analysis in classical Hodgkin lymphoma reveals new insights into the mechanisms underlying silencing of B cell-specific genes. Leukemia 2012, 26, 185-188. [CrossRef]

2. D'Alò, F.; Leone, G.; Hohaus, S.; Teofili, L.; Bozzoli, V.; Tisi, M.C.; Rufini, V.; Calcagni, M.L.; Voso, M.T. Response to 5-azacytidine in a patient with relapsed Hodgkin lymphoma and a thera-py-related myelodysplastic syndrome. Br. J. Haematol. 2011, 154, 141-143. [CrossRef]

3. Falchi, L.; Sawas, A.; Deng, C.; Amengual, J.E.; Colbourn, D.S.; Lichtenstein, E.A.; Khan, K.A.; Schwartz, L.H.; O'Connor, O.A. High rate of complete responses to immune checkpoint inhibitors in patients with relapsed or refractory Hodgkin lymphoma previously exposed to epigenetic therapy. J. Hematol. Oncol. 2016, 9, 1-4. [CrossRef]

4. Yuki, H.; Ueno, S.; Tatetsu, H.; Niiro, H.; Iino, T.; Endo, S.; Kawano, Y.; Komohara, Y.; Takeya, M.; Hata, H.; et al. 1 is a potent tumor suppressor in classical Hodgkin lymphoma cells. Blood 2013, 121, 962-970. [CrossRef] [PubMed]

5. Paczkowska, J.; Soloch, N.; Bodnar, M.; Kiwerska, K.; Janiszewska, J.; Vogt, J.; Domanowska, E.; Martin-Subero, J.I.; Ammerpohl, O.; Klapper, W.; et al. Expression of ELF1, a lymphoid ETS domain-containing transcription factor, is re-currently lost in classical Hodgkin lymphoma. Br. J. Haematol. 2019, 185, 79-88. [CrossRef] [PubMed]

6. Coffre, M.; Koralov, S.B. miRNAs in B Cell Development and Lymphomagenesis. Trends Mol. Med. 2017, 23, 721-736. [CrossRef] [PubMed]

7. Li, G.; So, A.Y.-L.; Sookram, R.; Wong, S.; Wang, J.K.; Ouyang, Y.; He, P.; Su, Y.; Casellas, R.; Baltimore, D. Epigenetic silencing of miR-125b is required for normal B-cell development. Blood 2018, 131, 1920-1930. [CrossRef]

8. Blume, J.; Ziętara, N.; Witzlau, K.; Liu, Y.; Sanchez, O.O.; Puchałka, J.; Winter, S.J.; Kunze-Schumacher, H.; Saran, N.; Düber, S.; et al. miR-191 modulates B-cell development and targets transcription factors E2A, Foxp1, and Egr1. Eur. J. Immunol. 2019, 49, 121-132. [CrossRef] [PubMed]

9. Arbore, G.; Henley, T.; Biggins, L.; Andrews, S.; Vigorito, E.; Turner, M.; Leyland, R. MicroRNA-155 is essential for the optimal proliferation and survival of plasmablast B cells. Life Sci. Alliance 2019, 2, e201800244. [CrossRef] [PubMed]

10. Brune, V.; Tiacci, E.; Pfeil, I.; Doring, C.; Eckerle, S.; van Noesel, C.J.; Klapper, W.; Falini, B.; von Heydebreck, A.; Metzler, D.; et al. Origin and pathogenesis of nodular lymphocyte-predominant Hodgkin lymphoma as re-vealed by global gene expression analysis. J. Exp. Med. 2008, 205, 2251-2268. [CrossRef]

11. Chomczynski, P. A reagent for the single-step simultaneous isolation of RNA, DNA and proteins from cell and tissue samples. Biotechniques 1993, 15, 532-534.

12. Mets, E.; Van Peer, G.; Van der Meulen, J.; Boice, M.; Taghon, T.; Goossens, S.; Mestdagh, P.; Benoit, Y.; De Moerloose, B.; Van Roy, N.; et al. MicroRNA-128-3p is a novel oncomiR targeting PHF6 in T-cell acute lympho-blastic leukemia. Haematologica 2014, 99, 1326-1333. [CrossRef] [PubMed]

13. Kluiver, J.; Slezak-Prochazka, I.; van den Berg, A. Studying microRNAs in lymphoma. Methods Mol. Biol. 2013, 971, 265-276. [PubMed] 
14. Bednarek, K.; Kiwerska, K.; Szaumkessel, M.; Bodnar, M.; Kostrzewska-Poczekaj, M.; Marszalek, A.; Janiszewska, J.; Bartochowska, A.; Jackowska, J.; Wierzbicka, M.; et al. Recurrent CDK1 overexpression in laryngeal squamous cell carcinoma. Tumor Biol. 2016, 37, 11115-11126. [CrossRef]

15. Paczkowska, J.; Janiszewska, J.; Bein, J.; Schneider, M.; Bednarek, K.; Ustaszewski, A.; Hartmann, S.; Hansmann, M.L.; Giefing, M. The Tumor Suppressive mir-148a Is Epigenetically Inactivated in Classical Hodg-kin Lymphoma. Cells 2020, 9, 2292. [CrossRef] [PubMed]

16. Torlakovic, E.; Tierens, A.; Dang, H.D.; Delabie, J. The Transcription Factor PU.1, Necessary for B-Cell Development Is Expressed in Lymphocyte Predominance, But Not Classical Hodgkin's Disease. Am. J. Pathol. 2001, 159, 1807-1814. [CrossRef]

17. Schmitz, R.; Hansmann, M.-L.; Bohle, V.; Martin-Subero, J.I.; Hartmann, S.; Mechtersheimer, G.; Klapper, W.; Vater, I.; Giefing, M.; Gesk, S.; et al. TNFAIP3(A20) is a tumor suppressor gene in Hodgkin lymphoma and primary mediastinal B cell lymphoma. J. Cell Biol. 2009, 185, i4. [CrossRef]

18. Kluiver, J.; Poppema, S.; de Jong, D.; Blokzijl, T.; Harms, G.; Jacobs, S.; Kroesen, B.J.; van den Berg, A. BIC and miR-155 are highly expressed in Hodgkin, primary mediastinal and diffuse large B cell lymphomas. J. Pathol. 2005, 207, 243-249. [CrossRef]

19. Navarro, A.; Gaya, A.; Martinez, A.; Urbano-Ispizua, A.; Pons, A.; Balagué, O.; Gel, B.; Abrisqueta, P.; Lopez-Guillermo, A.; Artells, R.; et al. MicroRNA expression profiling in classic Hodgkin lymphoma. Blood 2008, 111, 2825-2832. [CrossRef]

20. Van Vlierberghe, P.; De Weer, A.N.; Mestdagh, P.; Feys, T.; De Preter, K.; De Paepe, P.; Lambein, K.; Vandesompele, J.; Van Roy, N.; Verhasselt, B.; et al. Comparison of miRNA profiles of microdissected Hodg-kin/Reed-Sternberg cells and Hodgkin cell lines versus CD77+ B-cells reveals a distinct subset of differentially expressed miRNAs. Br. J. Haematol. 2009, 147, 686-690. [CrossRef]

21. Gibcus, J.H.; Tan, L.P.; Harms, G.; Schakel, R.N.; de Jong, D.; Blokzijl, T.; Möller, P.; Poppema, S.; Kroesen, B.J.; van den Berg, A. Hodgkin lymphoma cell lines are characterized by a specific miRNA expression profile. Neoplasia 2009, 11, 167-176. [CrossRef]

22. Sánchez-Espiridión, B.; Martín-Moreno, A.M.; Montalbán, C.; Figueroa, V.; Vega, F.; Younes, A.; Medeiros, L.J.; Alvés, F.J.; Canales, M.; Estévez, M.; et al. MicroRNA signatures and treatment response in patients with advanced classical Hodgkin lymphoma. Br. J. Haematol. 2013, 162, 336-347. [CrossRef]

23. Paczkowska, J.; Giefing, M. MicroRNA signature in classical Hodgkin lymphoma. J. Appl. Genet. 2021, 62, 281-288. [CrossRef]

24. Yuan, Y.; Niu, F.; Nolte, I.M.; Koerts, J.; De Jong, D.; Rutgers, B.; Osinga, J.; Azkanaz, M.; Terpstra, M.; Bystrykh, L.; et al. MicroRNA High Throughput Loss-of-Function Screening Reveals an Oncogenic Role for miR-21-5p in Hodgkin Lymphoma. Cell. Physiol. Biochem. 2018, 49, 144-159. [CrossRef]

25. Reed, S.M.; Hagen, J.; Muniz, V.P.; Rosean, T.R.; Borcherding, N.; Sciegienka, S.; Goeken, J.A.; Naumann, P.W.; Zhang, W.; Tompkins, V.S.; et al. NIAM-deficient mice are predisposed to the development of proliferative lesions in-cluding B-cell lymphomas. PLoS ONE 2014, 9, e112126. [CrossRef]

26. Slezak-Prochazka, I.; Kluiver, J.; De Jong, D.; Smigielska-Czepiel, K.; Kortman, G.; Winkle, M.; Rutgers, B.; Koerts, J.; Visser, L.; Diepstra, A.; et al. Inhibition of the miR-155 target NIAM phenocopies the growth promoting effect of miR-155 in B-cell lymphoma. Oncotarget 2015, 7, 2391-2400. [CrossRef] [PubMed]

27. Tie, Y.; Chen, C.; Yang, Y.; Qian, Z.; Yuan, H.; Wang, H.; Tang, H.; Peng, Y.; Du, X.; Liu, B. Upregulation of let-7f-5p promotes chemotherapeutic resistance in colorectal cancer by directly repressing several pro-apoptotic proteins. Oncol. Lett. 2018, 15, 8695-8702. [CrossRef] [PubMed]

28. Yuan, Y.; Kluiver, J.; Koerts, J.; De Jong, D.; Rutgers, B.; Razak, F.R.A.; Terpstra, M.; Plaat, B.E.; Nolte, I.M.; Diepstra, A.; et al. miR-24-3p Is Overexpressed in Hodgkin Lymphoma and Protects Hodgkin and Reed-Sternberg Cells from Apoptosis. Am. J. Pathol. 2017, 187, 1343-1355. [CrossRef] [PubMed]

29. Huang, X.; Zhou, X.; Wang, Z.; Li, F.; Liu, F.; Zhong, L.; Li, X.; Han, X.; Wu, Z.; Chen, S.; et al. CD99 triggers upregulation of miR-9-modulated PRDM1/BLIMP1 in Hodg-kin/Reed-Sternberg cells and induces redifferentiation. Int. J. Cancer 2012, 131, E382-E394. [CrossRef]

30. Leucci, E.; Zriwil, A.; Gregersen, L.H.; Jensen, K.T.; Obad, S.; Bellan, C.; Leoncini, L.; Kauppinen, S.; Lund, A. Inhibition of miR-9 de-represses HuR and DICER1 and impairs Hodgkin lymphoma tumour outgrowth in vivo. Oncogene 2012, 31, 5081-5089. [CrossRef]

31. Xin, H.; Wang, C.; Liu, Z. miR-196a-5p promotes metastasis of colorectal cancer via targeting IкB $\alpha$. BMC Cancer 2019, 19, 30. [CrossRef]

32. Danger, R.; Braza, F.; Giral, M.; Soulillou, J.-P.; Brouard, S. MicroRNAs, Major Players in B Cells Homeostasis and Function. Front. Immunol. 2014, 5. [CrossRef]

33. Robaina, M.C.; Mazzoccoli, L.; Klumb, C.E.; Klumb, E. Germinal Centre B Cell Functions and Lymphomagenesis: Circuits Involving MYC and MicroRNAs. Cells 2019, 8, 1365. [CrossRef] [PubMed]

34. Batista, C.R.; Li, S.K.H.; Xu, L.S.; Solomon, L.A.; DeKoter, R.P. PU.1 Regulates Ig Light Chain Transcription and Rearrangement in Pre-B Cells during B Cell Development. J. Immunol. 2017, 198, 1565-1574. [CrossRef] [PubMed]

35. Oettgen, P.; Akbarali, Y.; Boltax, J.; Best, J.; Kunsch, C.; Libermann, T.A. Characterization of NERF, a novel transcription factor re-lated to the Ets factor ELF-1. Mol. Cell Biol. 1996, 16, 5091-5106. [CrossRef]

36. Wertz, I.E.; O'rourke, K.M.; Zhou, H.; Eby, M.; Aravind, L.; Seshagiri, S.; Wu, P.; Wiesmann, C.; Baker, R.; Boone, D.L.; et al. De-ubiquitination and ubiquitin ligase domains of A20 downregulate NF-kappaB signalling. Nature 2004, 430, 694-699. [CrossRef] [PubMed] 
37. Guo, J.; Mei, H.; Sheng, Z.; Meng, Q.; Véniant, M.M.; Yin, H. Hsa-miRNA-23a-3p promotes atherogenesis in a novel mouse model of atherosclerosis. J. Lipid Res. 2020, 61, 1764-1775. [CrossRef]

38. Wei, C.; Zhang, R.; Cai, Q.; Gao, X.; Tong, F.; Dong, J.; Hu, Y.; Wu, G.; Dong, X. MicroRNA-330-3p promotes brain metastasis and epithelial-mesenchymal transition via GRIA3 in non-small cell lung cancer. Aging 2019, 11, 6734-6761. [CrossRef]

39. Mesci, A.; Huang, X.; Taeb, S.; Jahangiri, S.; Kim, Y.; Fokas, E.; Bruce, J.; Leong, H.; Liu, S.K. Targeting of CCBE1 by miR-330-3p in human breast cancer promotes metastasis. Br. J. Cancer 2017, 116, 1350-1357. [CrossRef]

40. Ye, Y.-P.; Wu, P.; Gu, C.-C.; Deng, D.-L.; Jiao, H.-L.; Li, T.-T.; Wang, S.-Y.; Wang, Y.-X.; Xiao, Z.-Y.; Wei, W.-T.; et al. miR-450b-5p induced by oncogenic KRAS is required for colorectal cancer progression. Oncotarget 2016, 7, 61312-61324. [CrossRef] 